\title{
Publisher Correction: Science that can change the world
}

Correction to: Nature Human Behaviour https://doi.org/10.1038/s41562-019-0642-2, published online 12 June 2019.

In the version of this article initially published, the editorial incorrectly stated that there was a 35\% increase in healthy purchases rather than health profile. The $35 \%$ improvement comprises a $31 \%$ reduction in junk food purchases and a $160 \%$ increase in healthy purchases (the increase in healthy purchases was large, in percentage terms, because the pre-intervention rate of healthy purchases was very small). The error has been corrected in all versions of the article.

Published online: 26 July 2019

https://doi.org/10.1038/s41562-019-0703-6 